VOL. 30 (1984), 321-334

\title{
ON SECOND DERIVATIVE ESTIMATES FOR EQUATIONS OF MONGE-AMPĖRE TYPE
}

NeIL S. TRUdinger AND JOHN I,E. URBAS

We derive interior second derivative estimates for solutions of equations of Monge-Amperre type which extend those of pogorelov for the case of affine boundary values. A key ingredient in our method is the existence of a strong solution of the homogeneous Monge-Ampère equation.

\section{Introduction}

Interior second derivative estimates for convex solutions of the Monge-Ampère equation $\operatorname{det} D^{2} u=g(x)$

were derived by Pogorelov [8], [9], under the restriction that the solution $u$ have affine boundary values. Here $g$ is a positive function in $C^{1,1}(\Omega)$ and $\Omega$ a convex domain in Euclidean $n$ space, $\mathbb{R}^{n}$. Pogorelov's method was subsequently extended to encompass MongeAmpere type equations of the form,

$$
\operatorname{det} D^{2} u=g(x, u, D u) \text {, }
$$

Received 12 April 1984.

Copyright Clearance Centre, Inc. Serial-fee code: 0004-9727/84 $\$ 2.00+0.00$ 
where $g$ is a positive function in $C^{1,1}\left(\Omega \times R \times R^{n}\right)$, in Lions [6,7] and Gilbarg and Trudinger [2]. A somewhat different approach, embracing less smooth functions $g$, was given by Ivochkina [3]. In this paper we establish interior estimates for solutions subject to $C^{1 / 1}$ boundary data. In particular we prove the following.

THEOREM 1. Let $\Omega$ be a $C^{1,1}$, uniformly convex domain in $\boldsymbol{R}^{n}$, $\varphi$ a function in $C^{1,1}(\bar{\Omega})$ and $g$ a positive function in $C^{1,1}\left(\Omega \times R \times \mathbb{R}^{n}\right)$. Then if $u$ is a convex classical solution of the Dirichlet problem

$$
\operatorname{det} D^{2} u=g(x, u, D u) \text { in } \Omega, u=\varphi \text { in } \partial \Omega \text {, }
$$

we have for any $\Omega^{\prime} \subset \subset \Omega$

$$
\sup _{\Omega}\left|D^{2} u\right| \leqslant C
$$

where $C$ is a constant depending only on $n, \Omega, \Omega,|\varphi|_{1,1 ; \Omega}, g,|u|_{0 ; \Omega}$ and the modulus of continuity of $u$ on $\partial \Omega$.

Our derivation of Theorem 1 rests on the following existence theorem for the homogeneous Monge-Ampère equation.

THEOREM 2. Let $\Omega$ be a $C^{1,1}$, uniformly convex domain in $\mathbb{R}^{n}$ and $\varphi \in C^{1 / 1}(\bar{\Omega})$. Then there exists a unique, convex solution $u \in C^{1,1}(\Omega) \cap C^{0,1}(\bar{\Omega})$ of the Dirichlet problem.

$$
\operatorname{det} D^{2} u=0 \text { in } \Omega, u=\varphi \text { in } \partial \Omega \text {. }
$$

Theorem 2 improves earlier work, in particular that of Rauch and Taylor [10], concerning the existence of generalized solutions of (1.5). As in [10], the solution $u$ is characterized as the lower boundary of the convex hull in $\mathbb{R}^{n+1}$ of the boundary manifold $(\partial \Omega, \varphi)$. We have also been informed that a result similar to Theorem 2 has been proved by Bedford and Taylor. The passage from Theorem 2 to Theorem 1 will be accomplished with the aid of the pogorelov method.

Theorems 1 and 2 are proved in sections 2 and 3 of this paper. Notation, unless otherwise indicated, will follow that of the book [2] . 


\section{The Homogeneous Equation}

In this section we will prove Theorem 2. The existence of a unique convex generalized solution of (1.5) was proved in [10] under the weaker hypotheses that $\Omega$ is bounded and strictly convex and $\varphi \in C^{0}(\bar{\Omega})$, so we need only prove the regularity assertion. Geometrically, the graph of $u$ is the lower boundary of the convex hull of graph $\left(\left.\varphi\right|_{\partial \Omega}\right)$.

To prove that $u \in C^{0,1}(\bar{\Omega})$ we can assume without loss of generality that $\varphi$ is convex, so that $\varphi$ is a lower barrier for $u$. Also, using the convexity of $u$, we have

$$
\frac{u(x)-u(y)}{|x-y|} \leqslant|D \varphi|_{0 ; \Omega}
$$

for all $y \in \partial \Omega$ and $x \in \Omega$. We thus obtain a global gradient bound for $u$.

It remains only to prove that $u \in C^{1,1}(\Omega)$. This will be carried out in the following lemmas. Let $M=\operatorname{graph}\left(\left.u\right|_{\Omega}\right), \partial M=\bar{M} \cap(\partial \Omega \times \mathbb{R})$, and for $E \subset \mathbb{R}^{n+1}$ let $\operatorname{conv}(E)$ denote the convex hull of $E$. If $x, y \in \mathbb{R}^{n+1},[x, y]$ denotes the closed line segment joining $x$ and $y$. LEMMA 2.1 Let $T$ be a supporting hyperplane of $M$ at $\xi \in M$. Then

$$
T \cap \bar{M}=\operatorname{conv}(T \cap \partial M)
$$

Proof. For convenience we assume that $u(\xi)=0$ and $T=\left\{x \in \mathbb{R}^{n+1}: x_{n+1}=0\right\}$. Then $T \cap \bar{M}=\{x \in \bar{\Omega}: u(x)=0\}$ and $T \cap \partial M=\{x \in \partial \Omega: u(x)=0\}$.

Suppose $y \in T \cap \bar{M}-\operatorname{conv}(T \cap \partial M)$. Then there is an $n-1$

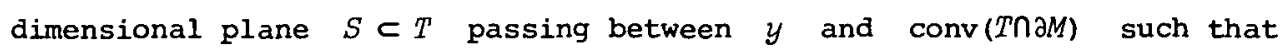
$d(y, S)>0$ and $d(S, \operatorname{conv}(T \cap \partial M))>0$. Let $S^{+}$and $S^{-}$denote the half spaces in $\mathbb{R}^{n}$ associated with $S$. We may assume that $S=\left\{x \in \mathbb{R}^{n}: x_{1}=0\right\}$ and $S^{-}=\left\{x \in \mathbb{R}^{n}: x_{2}<0\right\}$. Assume also that $\operatorname{conv}(T \cap \partial M) \subset S^{-}$. Then for some $\varepsilon>0$ we have $u>\varepsilon$ on $S^{+} \cap \partial \Omega$ and $u \leqslant 0$ on $S^{-} \cap \partial \Omega$. Hence for $\delta>0$ sufficiently small, $Q=\left\{x \in \mathbb{R}^{n+1}: \delta x_{1}-x_{n+1}=0\right\}$ is a hyperplane containing $S$, 
$y \in\left\{x \in \mathbb{R}^{n+1}: \delta x_{1}-x_{n+1}>0\right\}$ and $\operatorname{graph}\left(\left.\varphi\right|_{\partial \Omega}\right) \subset\left\{x \in \mathbb{R}^{n+1}: \delta x_{1}-x_{n+1}<0\right\}$. Thus $y \notin \operatorname{conv}\left(g r a p h\left(\left.\varphi\right|_{\partial \Omega}\right)\right)$, which is a contradiction.

LEMMA 2.2 For each $\xi \in M$ there are $\zeta_{1} \in \partial M$ and $\zeta_{2} \in M$ such that $\xi \in\left[\zeta_{1}, \zeta_{2}\right] \subset \bar{M}$ and

$$
\left|\xi-\zeta_{2}\right| \geqslant \frac{1}{2 n}\left|\zeta_{1}-\zeta_{2}\right|
$$

Proof. Let $T$ be a supporting hyperplane of $M$ at $\xi$. Then by Lemma 2.1 we may choose $n+1$ points $\xi_{1}, \ldots \xi_{n+1}$ in $T \cap \partial M$ such that $\xi \in \operatorname{conv}\left\{\xi_{i}\right\}_{i=1}^{n+1}$. From these points we may choose $k$ points, say $\xi_{1}, \ldots, \xi_{k}$ such that $\xi \in \operatorname{int} \operatorname{conv}\left\{\xi_{i}\right\}_{i=1}^{k}$, where int $\operatorname{conv}\left\{\xi_{i}\right\}_{i=1}^{k}$ denotes the $k-1$ dimensional interior of the simplex $P=\operatorname{conv}\left\{\xi_{i}\right\}_{i=1}^{k}$.

Let $n_{i}$ be the unique point in $\partial P$ such that $\xi \in\left[\xi_{i}, n_{i}\right]$. Since $\xi \in$ int $P$, we have $\eta_{i} \in$ int $F_{i}$ for some face $F_{i}$ of $P$, and no two $n_{i}$ lie in the same face. We will show that for some $i$ $\zeta_{1}=\xi_{i}$ and $\zeta_{2}=\eta_{i}$ satisfy the conclusion of the lemma.

Suppose this is not the case. Then

$$
\text { 、 } \xi \in G_{i}=\left\{x \in P: d\left(x, F_{i}\right)<\frac{1}{2 n} d\left(\xi_{i}, F_{i}\right)\right\}
$$

for all $i=1, \ldots, k$. For each $j=1, \ldots, k, n_{i \neq j} G_{i}$ is a $k-1$ dimensional parallelogram with side lengths $\frac{1}{2 n} d\left(\xi_{i}, F_{i}\right)$ for $i \neq j$, and $\xi_{j} \in \underset{i \neq j}{n} F_{i} \subset \bigcap_{i \neq j} G_{i}$ is a vertex of this parallelogram. Thus

$$
\left|\xi-\xi_{j}\right|<\frac{1}{2 n} \sum_{i \neq j} d\left(\xi_{i}, F_{i}\right)
$$

and hence

$$
\begin{aligned}
d\left(\xi_{j}, F_{j}\right) & \leqslant\left|\xi-\xi_{j}\right|+d\left(\xi, F_{j}\right) \\
& \leqslant \frac{1}{2 n} \sum_{i=1}^{k} d\left(\xi_{i}, F_{i}\right) .
\end{aligned}
$$


Sumning over $j$ from 1 to $k$ we obtain a contradiction, so the lemma is proved.

LEMMA 2.3 Let $x_{0} \in \boldsymbol{s}$ and $\xi_{0}=\left(x_{0}, u\left(x_{0}\right)\right)$. Then if $x \in B=\left\{x \in \Omega:\left|x-x_{0}\right|<\frac{1}{\sigma n} d\left(x_{0}, \partial \Omega\right)\right\}$, we have

$$
u(x) \leqslant u\left(x_{0}\right)+\operatorname{Du}\left(x_{0}\right) \cdot\left(x-x_{0}\right)+C\left|x-x_{0}\right|^{2},
$$

where $C$ depends only on $n,|\varphi|_{1,1 ; \Omega}, \operatorname{diam} \Omega, d\left(x_{0}, \partial \Omega\right)$ and $a$ positive lower bound $R$ on the principal radii of curvature of $\partial \Omega$.

Proof. Let $\zeta_{1} \in \partial M$ and $\zeta_{2} \in M$ be the points associated with $\xi_{0}$ as in Lemma 2.2. Assume for convenience that $\zeta_{2}=(0,0)$. Let $\psi: \bar{\Omega}-\{0\} \rightarrow \partial \Omega$ be the radial retraction. Then we clearly have for $x \in \bar{\Omega}-\{0\}$,

$$
|x| \leqslant|\psi(x)|
$$

and by Lemma 2.2 ,

$$
d\left(x_{0}, \partial \Omega\right) \leqslant\left|\psi\left(x_{0}\right)\right| \leqslant 2 n\left|x_{0}\right|
$$

If $x \in B^{\prime}=\left\{x \in \Omega:\left|x-x_{0}\right|<\frac{1}{4 n} d\left(x_{0}, \partial \Omega\right)\right\}$, we have

$$
\begin{aligned}
d(x, \partial \Omega) & \geqslant d\left(x_{0}, \partial \Omega\right)-\left|x-x_{0}\right| \\
& \leqslant\left(1-\frac{1}{4 n}\right) d\left(x_{0}, \partial \Omega\right),
\end{aligned}
$$

and also, from (2.5),

$$
\begin{aligned}
\frac{1}{|x|} & \leqslant \frac{1}{\left|x_{0}\right|-\left|x-x_{0}\right|} \\
& \leqslant \frac{4 n}{d\left(x_{0}, \partial \Omega\right)} .
\end{aligned}
$$

Assuming initially that $\partial \Omega \in C^{2}$ and $\varphi \in C^{2}(\bar{\Omega})$ we define $w: \bar{\Omega} \rightarrow \boldsymbol{R}$ by

$$
\omega(x)= \begin{cases}\frac{|x|}{|\psi(x)|} \varphi(\psi(x)) & \text { if } x \neq 0 \\ 0 & \text { if } x=0 .\end{cases}
$$


Geometrically, the graph of $w$ is the cone with base graph $\left(\left.\varphi\right|_{\partial \Omega}\right)$ and vertex $(0,0)$. Clearly, $w \in C^{2}(\bar{\Omega}-\{0\})$, and $u \leqslant w$ in $\Omega$, by the convexity of $u$. Consequently the graphs of $u$ and $w$ are tangent at $x_{0}$ whence $u$ is differentiable there with $D u(x)=D w\left(x_{0}\right)$. Furthermore, differentiating (2.8) twice, and using (2.4) and (2.7), we obtain

$$
\left|D^{2} w\right|_{0 ; B^{\prime}} \leqslant C\left(n,|\varphi, \psi|_{\left.2 ; B^{\prime}, d\left(x_{0}, \partial \Omega\right)\right)}\right.
$$

We now proceed to obtain a bound for $|\psi|_{2 ; B}$, clearly, we have $|\psi|_{0 ; B}, \leqslant \operatorname{aiam} \Omega$. To obtain derivative bounds, it is convenient to use polar coordinates. We write

$$
\psi(x)=\tilde{\psi}\left(\theta, \ldots, \theta_{n-1}\right)
$$

where $\theta_{1}, \ldots, \theta_{n-1}$ are the angular variables. Then we obtain in $\vec{\Omega}-\{0\}$,

$$
D_{i} \psi=\sum_{k=1}^{n-1} D_{\theta_{k}} \tilde{\psi} D_{i} \theta_{k}
$$

and

$$
D_{i j} \psi=\sum_{k, \ell=1}^{n-1} D_{\theta_{k} \theta_{\ell}} \tilde{\psi}_{i} D_{i} D_{j} \theta_{\ell}+\sum_{k=1}^{n-1} D_{\theta_{k}} \tilde{\psi} D_{i, j} \theta_{k} \cdot
$$

Let $\nu$ be the outer unit normal to $\partial \Omega$ at $\psi(x)$, and $T$ the tangent $n-1$ plane to $\partial \Omega$ at $\psi(x)$. Then

$$
\frac{\psi(x) \cdot \nu}{|\psi(x)|}=\frac{d(0, T)}{|\psi(x)|} \geqslant \frac{d(x, \partial \Omega)}{\operatorname{diam} \Omega}
$$

Using (2.6), (2.7) and (2.13) we obtain from (2.11) the estimate

$$
|D \psi|_{0 ; B^{\prime}} \leqslant C\left(n, \text { diam } \Omega, d\left(x_{0}, \partial \Omega\right)\right),
$$

and from (2.13), also using

$$
\left|D_{\theta_{k} \theta_{\ell}} \tilde{\psi}\right|_{0 ; B}, \leqslant C\left(n, R, d\left(x_{0}, \partial \Omega\right)\right)
$$

we obtain 


$$
\left|D^{2} \psi\right|_{0 ; B^{\prime}} \leqslant C\left(n, R, \operatorname{diam} \Omega, d\left(x_{0}, \partial \Omega\right)\right)
$$

Thus we have a bound for $|\psi|_{2 ; B}$, and hence

$$
\left|D^{2} w\right|_{0 ; B} \leqslant C
$$

where $C$ depends on $n, R, \operatorname{diam} \Omega, d\left(x_{0}, \partial \Omega\right)$ and $|\varphi|_{2 ; \Omega^{*}}$

Now let $\left\{\Omega_{m}\right\}$ be an increasing sequence of $C^{2}$ uniformly convex subdomains of $\Omega, \cup \Omega_{m}=\Omega$, and $\left\{\varphi_{m}\right\} \subset C^{2}(\bar{\Omega})$ a sequence of functions converging in $C^{1, \alpha}(\bar{\Omega})$ to $\varphi, \alpha<1$, and satisfying $\left|\varphi_{m}\right|_{2 ; \Omega} \leqslant 2|\varphi|_{1,1 ; \Omega}$. Let $w_{m}: \bar{\Omega} \rightarrow R$ be the function defined by

$$
w_{m}(x)= \begin{cases}\frac{|x|}{\left|\Psi_{m}(x)\right|} \varphi_{m}\left(\psi_{m}(x)\right) & \text { if } x \neq 0 \\ 0 & \text { if } x=0 ，\end{cases}
$$

where $\psi_{m}: \bar{\Omega}-\{0\} \rightarrow \partial \Omega_{m}$ is the radial retraction. For $m$ sufficiently large, we then have uniform bounds for $\left|D^{2} w_{m}\right|_{0 ; B}$ where $B=\left\{x \in \Omega:\left|x-x_{0}\right|<\frac{1}{6 n} d\left(x_{0}, \partial \Omega\right)\right\}$, and therefore since $w_{m}$ converges to $w$ in $C^{1, \alpha}(\bar{\Omega}-\{0\}), \alpha<1$, we obtain $w \in C^{1,1}(\bar{\Omega}-\{0\})$ and

$$
[D w]_{1: B} \leqslant C
$$

where $C$ depends only on $n, R$, dian $\Omega, d\left(x_{0}, \partial \Omega\right)$ and $|\varphi|_{1,1 ; \Omega}$.

We can now obtain the conclusion of the lemma by using Taylor's theorem and the fact that $D w\left(x_{0}\right)=D u\left(x_{0}\right)$.

We are now ready to complete the proof of Theorem 2. Let $\gamma$ be a unit vector in $\boldsymbol{R}^{n}$, and form the second order difference quotient of $u$ with respect to $\gamma$

$$
\Delta_{\gamma \gamma}^{h} u(x)=\frac{1}{h^{2}}\{u(x+h \gamma)+u(x-h \gamma)-2 u(x)\} .
$$

Then for each $\Omega^{\prime} \subset \subset \Omega$, we have, from Lemma 2.3, for all $h>0$ sufficiently small, 


$$
\left|\Delta_{\gamma \gamma^{\prime}}^{h} u\right|_{L^{\infty}\left(\Omega^{\prime}\right)} \leqslant C
$$

Hence we can extract a subsequence $\left\{h_{m}\right\}$ converging to zero such that ${ }_{\Delta}^{{ }^{h}}{ }_{m_{\gamma}} u$ converges in the weak ${ }^{\star}$ topology on $L^{\infty}\left(\Omega^{\prime}\right)$ to a function $w_{\gamma} \in \cdot L^{\infty}\left(\Omega^{\prime}\right)$. Thus it follows that the distributional derivative $D_{\gamma \gamma}^{u}$ is representable by a function in $L_{10 c}^{\infty}(\Omega)$. Since $\gamma$ is an arbitrary direction, we conclude that $u \in C^{1,1}(\Omega)$, and for each $\Omega^{\prime} \subset \subset \Omega$,

$$
[D u]_{1 ; \Omega} \leqslant C,
$$

where $C$ depends only on $n, R$, diam $\Omega, d\left(\Omega^{\prime}, \partial \Omega\right)$ and $|\varphi|_{1,1 ; \Omega}$.

\section{Second Derivative Estimates}

In this section we will prove Theorem 1 . Writing the equation (1.2) in the form

$$
F\left(D^{2} u\right)=\log \operatorname{det} D^{2} u=f(x, u, D u)
$$

where $f=\log g$, we have

$$
\begin{gathered}
F_{i j}=u^{i j} \\
F_{i j, k l}=-u^{i k} u^{j l}=-F_{i k} F_{j l} .
\end{gathered}
$$

where $\left[u^{i j}\right]$ denotes the inverse of $D^{2} u$.

Next, we note that any pure second derivative $D_{\gamma \gamma} u$ of a solution $u \in C^{4}(\Omega)$ of (3.1) satisfies the equation

$$
F_{i j} D_{i j \gamma \gamma} u=\dot{F}_{i k} F_{j \ell} D_{i j \gamma} u D_{k \ell \gamma} u+D_{\gamma \gamma} f
$$

Since $u$ is convex, we have $D_{\gamma \gamma} u \geqslant 0$, so we need only estimate $D_{\gamma \gamma} u$ from above.

$$
\begin{aligned}
& \text { We now fix } \Omega^{\prime} \subset \subset \Omega \text { and set } \delta=d\left(\Omega^{\prime}, \partial \Omega\right), \\
& \Omega^{\prime \prime}=\{x \in \Omega: d(x, \partial \Omega)>\delta / 2\} \text { and } \Omega^{\prime \prime \prime}=\{x \in \Omega: d(x, \partial \Omega)>\delta / 4\} \text {. We }
\end{aligned}
$$


first prove a lower bound for ${ }_{\Omega^{\prime \prime}}(v-u)$, where $v$ is the convex solution of the Dirichlet problem (1.5). Let $x_{0} \in \Omega^{\prime \prime}$ and for $\sigma>0$ set

$$
\psi(x)=\psi_{\sigma}(x)=-\sigma\left((\delta / 4)^{2}-\left|x-x_{0}\right|^{2}\right) .
$$

We have $D_{i j} \psi=2 \sigma \delta_{i j}$, so

$$
\begin{gathered}
\operatorname{det}\left(D^{2} v+D^{2} \psi\right) \leqslant C_{1}(n) \sum_{k=1}^{n} \sigma^{k} M^{n-k}, \\
M=\sup _{\Omega^{\prime}}\left|D^{2} v\right| .
\end{gathered}
$$

where

We also have

$$
\sup _{\Omega i n}|D u| \leqslant 8 \delta^{-1}|u|_{0 ; \Omega}
$$

and hence

$$
\inf _{\Omega \cdot m} g(x, u, D u) \geqslant \lambda>0,
$$

where $\lambda$ is a constant depending only on $|u|_{0 ; \Omega}, \delta$ and $g$. Choosing $\sigma>0$ so small that

$$
c_{1}(n) \sum_{k=1}^{n} \sigma^{k} M^{n k} \leqslant \lambda .
$$

and using the comparison principle, we obtain $v-u \geqslant-\psi$ in $B_{\delta / 4}\left(x_{0}\right)$, and hence

$$
\inf _{\Omega^{\prime \prime}}(v-u) \geqslant \sigma\left(\frac{\delta}{4}\right)^{2} .
$$

We now consider the function

$$
n=n_{\varepsilon}=(v-u-\varepsilon)^{+}
$$

in the set $\Omega_{\varepsilon}=\{x \in \Omega: \eta(x)>0\}$. Setting $\varepsilon=\frac{1}{2} \sigma(\delta / 4)^{2}$, we have $\Omega^{\prime \prime} \subset \subset \Omega_{\varepsilon}$, and we can estimate $d\left(\Omega_{\varepsilon}, \partial \Omega\right)$ from below in terms of $\varepsilon$, $|D v|_{0 ; \Omega}$ and a modulus of continuity for $u$. We then have

$$
\sup _{\varepsilon}|D u| \leqslant C_{2}
$$


and hence also

$$
\inf _{\varepsilon} g(x, u, D u) \geqslant C_{3}>0
$$

and

$$
\sup _{\Omega}\left(|D f(x, u, D u)|+\left|D^{2} f(x, u, D u)\right|\right) \leqslant C_{4} .
$$

where $C_{2}, C_{3}$ and $C_{4}$ depend on $n,|u|_{0 ; \Omega},|\varphi|_{1,1 ; \Omega}, \Omega, d\left(\Omega^{\prime}, \partial \Omega\right)$, $g$ and a modulus of continuity for $u$. Here we have used the estimates of the previous section to remove the dependence on $v$.

We now consider, in the set $\Omega_{\varepsilon}$, the function

$$
w=\eta h(D u) D_{\gamma \gamma} u
$$

where $h \in C^{2}\left(\mathbb{R}^{n}\right)$ is a positive function to be chosen. We then have

$$
\begin{aligned}
& \frac{D_{i} w}{w}=\frac{D_{i} \eta}{n}+(\log h) p_{k} D_{i k^{u}}+\frac{D_{i \gamma \gamma^{u}}}{D_{\gamma \gamma}{ }^{u}}, \\
& \frac{D_{i j} \omega}{w}=\frac{D_{i} \omega D_{j} \omega}{\omega^{2}}+\frac{D_{i j}{ }^{n}}{n}-\frac{D_{i}{ }^{D} D_{j}}{n^{2}}+ \\
& (\log h)_{p_{k} p_{\ell}}{ }^{D_{i k} u D_{j \ell} u+(\log h)} p_{k}{ }_{i j k} u+ \\
& \frac{D_{i j \gamma \gamma^{u}}}{D_{\gamma \gamma} u}-\frac{D_{i \gamma \gamma} u D_{j \gamma \gamma} u}{\left(D_{\gamma \gamma} u\right)^{2}}
\end{aligned}
$$

Using (3.3), we obtain

$$
\begin{aligned}
& (\eta h)^{-1} F_{i j} D_{i j} \omega \geqslant D_{\gamma \gamma} u\left\{\frac{F_{i j} D_{i j}{ }^{n}}{n}-\frac{F_{i j} D_{i}{ }^{n} D_{j}{ }^{n}}{n^{2}}+\right. \\
& \left.{ }^{(\log h)}{ }_{p_{k} p_{\ell}{ }^{{ }}{ }_{i j}{ }^{D} i k^{u D}} \ell^{u+(\log h)}{ }_{p_{k}}{ }^{E_{i j}}{ }_{i j k}{ }^{u}\right\}+
\end{aligned}
$$

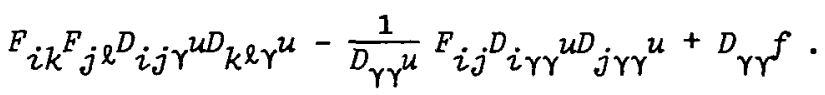


An obvious choice for $h$ is

$$
h(p)=\exp \left(\beta|p|^{2} / 2\right), \beta>0,
$$

so that

$$
{ }^{(\log h)} p_{k}=B p_{k},(\log h){ }_{p_{k} p_{\ell}}=B \delta_{k \ell} .
$$

and hence

$$
{ }^{(\log h)_{p_{k} p_{\ell}}{ }^{F} i j{ }^{D} k^{u D_{j \ell}} u=\beta F_{i j}{ }_{i k} u D_{j k} u=\beta \Delta u}
$$

by (3.2) .

Next, making use of the estimates $(3.5),(3.6)$ and $(3.7)$, we obtain

$$
\begin{aligned}
& { }^{D_{\gamma}}{ }^{u(\log h)}{ }_{p_{k}}{ }_{i j} D_{i j k} u+D_{\gamma \gamma} f=B D_{k} u D_{\gamma \gamma} u\left(f_{x_{k}}+f_{z} D_{k} u+f_{p_{i}} D_{i k} u\right)+ \\
& f_{\gamma \gamma}+2 f_{\gamma z} D_{\gamma} u+2 f_{\gamma p_{i}} D_{i \gamma} u+f_{z Z}\left(D_{\gamma} u\right)^{2}+ \\
& 2 f_{z p_{i}{ }^{D}} u D_{i \gamma} u+f_{p_{i} p_{j}}{ }_{i \gamma} u{ }_{j \gamma}{ }^{u}+f_{z}{ }^{D} \gamma \gamma^{u}+f_{p_{i}}{ }_{i \gamma \gamma} u \\
& \geqslant g_{p_{i}}\left(\frac{D_{i} w}{w}-\frac{D_{i} \eta}{n}\right) D_{\gamma \gamma} u-C_{5}\left\{1+\left|D^{2} u\right|^{2}+B\left(1+\left|D^{2} u\right|\right)\right\} \text {, }
\end{aligned}
$$

where $C_{5}$ depends on the same quantities as $C_{2}, C_{3}$ and $C_{4}$.

In order to handle the other terms in $(3.8)$ we regard $w=w(x, \gamma)$ as a function on $\Omega_{\varepsilon} \times \partial B_{1}(0)$ and suppose $w$ takes a maximum value at a point $y \in \Omega_{\varepsilon}$ and direction $\gamma$. The derivative $D_{\gamma \gamma} u(y)$ is then the maximum eigenvalue of the Hessian $D^{2} u(y)$ and by a rotation of coordinates we can assume that $D^{2} u(y)$ is in diagonal form with $\gamma$ a coordinate direction. We now have

$$
\begin{aligned}
F_{i j} D_{i j}{ }^{n} & =F_{i j} D_{i j} v-F_{i j} D_{i j}^{u} \\
& \geqslant-n .
\end{aligned}
$$

Furthermore, since $D w(y)=0$, we have 


$$
\begin{aligned}
& F_{i j} \frac{D_{i} n D_{j} n}{n^{2}}=\frac{\Sigma E_{i i}\left|D_{i} n\right|^{2}}{n^{2}} \\
& =\sum_{i \neq \gamma} F_{i i}\left(B D_{k} u D_{i k^{u}} u+\frac{D_{i \gamma \gamma^{u}}}{D_{\gamma \gamma} u}\right)^{2}+\frac{\left|D_{\gamma} n\right|^{2}}{n^{2} D_{\gamma \gamma} u} \\
& \leqslant \frac{\left|D_{\gamma} n\right|^{2}}{n^{2} D_{\gamma \gamma^{u}}}+\sum_{i \neq \gamma} E_{i i}\left(\frac{D_{i \gamma \gamma} u}{D_{\gamma \gamma} u}\right)^{2}-2 \beta \sum_{i \neq \gamma} \frac{D_{i} D_{i} u}{n}
\end{aligned}
$$

at the point $y$. Also,

$$
\begin{aligned}
& \frac{1}{D_{\gamma \gamma} u}\left\{\sum_{i \neq \gamma} F_{i i}\left(D_{i \gamma \gamma} u\right)^{2}+F_{i j} D_{i \gamma \gamma} u D_{j \gamma \gamma} u\right\} \\
& =\sum_{i \neq \gamma} F_{\gamma \gamma} F_{i i}\left(D_{i \gamma \gamma} u\right)^{2}+\sum_{i=1}^{n} F_{\gamma \gamma} F_{i i}{ }^{\left(D_{i \gamma \gamma} u\right)^{2}} \\
& \leqslant \sum_{i, j=1}^{n} F_{i i} F_{j j}\left(D_{i j \gamma} u\right)^{2} \\
& =F_{i k} F_{j l} D_{i j \gamma} u D_{k \ell \gamma} u
\end{aligned}
$$

at $y$, by virtue of our choice of coordinates. Taking the above estimates into account in (3.8), and then choosing $\beta$ sufficiently large, we obtain by virtue of the strong maximum principle, ([2], Theorem 9.6)

$$
D_{\gamma \gamma} u(y) \leqslant C_{6}\left(1+\frac{1}{\eta(y)}\right) \text {, }
$$

and hence

$$
\sup _{\Omega_{\varepsilon}} w \leqslant C_{7}
$$

where $C_{6}$ and $C_{7}$ depend on the same quantities as $C_{2}, C_{3}, C_{4}$ and $C_{5}$. Making use of $(3.4)$, we obtain $(1.4)$ as required.

Remarks (i) When $\varphi$ vanishes on $\partial \Omega$ we can assume that $\Omega$ is an arbitrary bounded convex domain in $\boldsymbol{R}^{n}$. 
(ii) Using Theorem 1, we may infer existence theorems for the Dirichlet problem (1.3) by direct approximation from the globally smooth case treated by Caffarelli, Nirenberg and spruck [1], Krylov [5] and Ivochkina [4]. In particular we may obtain the results of [6], [7], [11] in this way, without having to invoke regularity considerations for generalized solutions.

(iii) Note that we only need $u \in W^{4}, n(\Omega) \cap C^{3}(\Omega) \cap C^{0}(\bar{\Omega})$ in the proof of Theorem 1; the assumptions on $g$ automatically ensure such regularity for classical solutions $u \in C^{2}(\Omega) \cap C^{0}(\bar{\Omega}),([2]$, Lemma 17.16).

\section{References}

[1] L. Caffarelli, L. Nirenberg, J. Spruck, "The Dirichlet problem for nonlinear second order elliptic equations, I. Monge-Ampère equation. Comm. Pure Appl. Math. 37 (1984), 369-402.

[2] D. Gilbarg, N.S. Trudinger, EZZiptic partial differential equations of second order, 2nd edition (Springer-Verlag, Berlin, Heidelberg, New York, Tokyo, 1983).

[3] N.M. Ivochkina, "Construction of a priori bounds for convex solutions on the Monge-Amperre equation by integral methods", Ukrain. Math. J. 30 (1978), 32-38.

[4] N.M. Ivochkina, "Classical solvability of the Dirichlet problem for the Monge-Ampère equation", Zap. Naučn. Sem. Leningrad, Otdel. Mat. Inst. Steklov. (LOMI) 131 (1983) 72-79.

[5] N.V. Krylov, "Boundedly inhomogeneous elliptic and parabolic equations in domains", Izvestia Akad. Nauk. SSSR, 47, (1983), 75-108.

[6] P.L. Lions, "Sur les equations de Monge-Ampère I", Manuscripta Math. 41 (1983), 1-43.

[7] P.L. Lions, "Sur les equations de Monge-Ampère II" Arch. Rat. Mech. Anal. (to appear).

[8] A.V. Pogorelov, "On the regularity of generalized solutions of the equation $\operatorname{det}\left(\partial^{2} u / \partial x_{i} \partial x_{j}\right)=\varphi\left(x_{1}, \ldots, x_{n}\right)>0 "$, Dokz. Akad. Nauk SSSR, 200 (1971), 1436-1440. 
N.S. Trudinger and J.I.E. Urbas

[9] A.V. Pogorelov, The Minkowski multidimensional problem, (wiley, New York 1978).

[10] J. Rauch, B.A. Taylor, "The Dirichlet problem for the multidimensional Monge-Ampère equation", Rocky Mountain J. Math. 7 (1977), 345-364.

[11] N.S. Trudinger, J.I.E. Urbas, "The Dirichlet problem for the equation of prescribed Gauss curvature", Bull. Austral. Math. Soc. 28 (1983), 217-231.

Centre for Mathematical Analysis

Australian National University

G.P.O. Box 4

Canberra A.C.T. 2601

Australia. 